\title{
An Evaluation of Changeability of Parameters Describing Abbot Curve during a Wavelet De- composition Process
}

Jozef Zajac ${ }^{1}$, Włodzimierz Makieła ${ }^{2}$, Krzysztof Stępień ${ }^{2}$, Damian Gogolewski

${ }^{1)}$ Technical University of Košice, Faculty of Manufacturing Technologies. E-mail: jozef.zajac@tuke.sk

${ }^{2)}$ Kielce University of Technology, Faculty of Mechatronics and Machine Design. E-amil: wmakiela@tu.kielce.pl, kstepien@tu.kielce.pl, dgogolewski@tu.kielce.pl

Results of signals analysis was done using wavelet transform, which have different curves of wavelets and depend on the basic wavelet, which were applied. Thus, it is sometimes not possible to conduct a wavelet transform of a given profile with the use of any basic wavelets and to obtain results that are similar to the measured signal. The aim of this was work was an optimization of a basic wavelet selection used for an analysis of surface roughness. In the work an analysis of Abbot-Firestone curve parameters was performed on subsequent decomposition levels and for various basic wavelets.

Keywords: wavelet transform, Abbot-Firestone curve, decomposition

\section{References}

[1] S. ADAMCZAK. (2008). Pomiary geometryczne powierzchni, WNT, Warszawa.

[2] S. ADAMCZAK, W. MAKIEŁA, K. STĘPIEŃ. (2010). Investing advantages and disadvantages of the analysis of a geometrical surface structure with the use of Fourier and wavelet transform. In: Metrology and Measurement System, Vol. XVII, pp. 233-244.

[3] P. AUGUSTYNIAK. (2003). Transformacje falkowe w zastosowaniach elektrodiagnostycznych. Kraków.

[4] Z. HUMIENN. (2004). Specyfikacje geometrii wyrobów (GPS). WNT, Warszawa.

[5] W. JAKUBIEC, J. MALINOWSKI. (2004). Metrologia wielkości geometrycznych. WNT, Warszawa.

[6] W. MAKIEŁA, K. STĘPIEŃ. (2010). Ocena wpływu metodyki doboru falki bazowej na analizę falkową zarysów nierówności powierzchni. In: Pomiary Automatyka Kontrola, Vol. 1, pp. 32-34.

[7] W. MAKIELA, K. STĘPIEŃ. (2012). Evaluation of flatness profiles by means of wavelet transform, In: Manufacturing and Industrial Engineering

[8] M. MISITI, Y. MISITI, G. OPPENHEIM, J. M. POGGI. (2007). Wavelet Toolbox 4 - User's Guide The MathWorks.

[9] K. E. OCZOŚ. (2003). Struktura geometryczna powierzchn. Rzeszów.

[10] M. WIECZOROWSKI. (2009). Wykorzystanie analizy topograficznej w pomiarach nierówności powierzchni. Poznań.

[11] A. ZAWADA-TOMKIEWICZ. (2009) Dekompozycja falkowa profilu powierzchni obrobionej po toczeniu. In: Pomiary Automatyka Kontrola, Vol.: 4, pp. 243-246.

[12] A. ZAWADA-TOMKIEWICZ. (2010). Zastosowanie dyskretnej transformaty falkowej do redukcji zakłóceń w obrazie powierzchni obrobionej. In: Zeszyty naukowe politechniki Poznańskiej - Budowa Maszyn i Zarządzanie produkcją, Vol. 13, pp. 173-178.

[13] A. ZAWADA-TOMKIEWICZ. (2012), Teoretyczne i doświadczalne podstawy monitorowania procesu toczenia z wykorzystaniem informacji o cechach stereometrycznych obrobionej powierzchni. Koszalin.

[14] Standard PN-ISO 13565-2:1999: Specyfikacje geometrii wyrobów - Struktura geometryczna powierzchni - Metoda profilowa - Powierzchnie o warstwowych właściwościach funkcjonalnych - Opis wysokości za pomocą linearyzacji krzywej udziału materiałowego

[15] M. NOVAK., H. KASUGA., H. OHMORI. (2013). Differences at the Surface Roughness by the ELID and Grinding Technology. In: Manufacturing technology, Vol.: 13, No. 2, pp. $210-215$

[16] M. NOVAK. (2012). Surfaces with high precision of roughness after grinding. In: Manufacturing technology, Vol.: 12, No. 12 , pp. 66-70. 